\title{
An Identification of Magnetizing Current Phenomenon in Steady State Condition
}

\author{
Meenakshi Sarswat, Lokesh Varshney
}

\begin{abstract}
This document is basically based on the magnetizing current phenomenon of transformer. In this paper we will see how the magnetizing current behave when the core material has linear and non-linear characteristics.
\end{abstract}

Keyword: Magnetization, Hysteresis, Oscillating Neutral, Sinusoidal

\section{INTRODUCTION}

\section{A. Magnetizing Curve}

At no load condition, Supply is connected on primary side of transformer, that current flow in primary is called magnetizing current. Another name is exciting current, whereas creates magnetic field in core and provides energy for no-load power losses in core.

Figure 1 shows the magnetization and demagnetization of a core under AC power. The curve on the left side is called the hysteresis curve of the magnetic material which represents the loss in magnetization and demagnetization of the core.

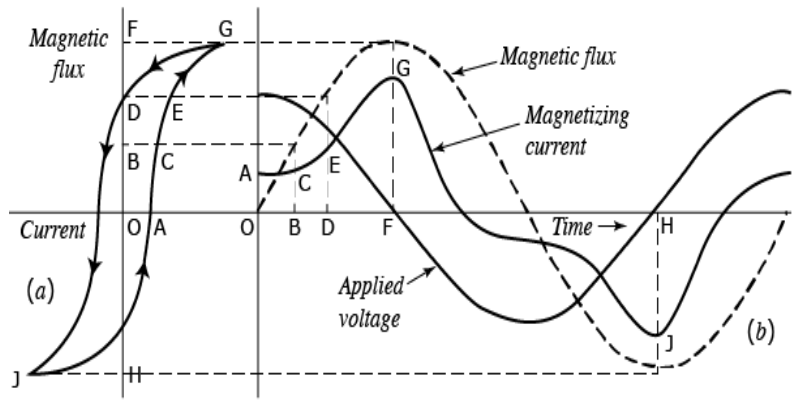

Waveform of magnetizing current.

Fig. 1.Magnetization and demagnetization of of any core under AC power.

Hysteresis

Heat is produced when the friction occurs between the magnetizing force and molecules of the magnet known as hysteresis loss.

When internal magnetic field is applied, molecules of magnetic field are aligned with one side, and when the magnetic field is inverted, inner magnetic collision of cells opposes magnetic field leading to magnetic hysteresis. To wipe out or overcome this inner friction known as residual magnetism.

Manuscript received on May 10, 2021.

Revised Manuscript received on May 18, 2021.

Manuscript published on June 30, 2021.

* Corresponding Author

Meenakshi Sarswat*, School of Electrical, Electronics \& Communication Engineering, Galgotias University Greater Noida (U.P), India. Email: meenakshi.mataray@gmail.com

Lokesh Varshney, School of Electrical, Electronics \& Communication Engineering, Galgotias University Greater Noida (U.P), India. Email: lokesh.varshney2021@gmail.com

(C) The Authors. Published by Blue Eyes Intelligence Engineering and Sciences Publication (BEIESP). This is an open access article under the CC BY-NC-ND license (http://creativecommons.org/licenses/by-nc-nd/4.0/)

\section{MAGNETISATION CURRENT}

When the ac power source is connected to the primary transformer, the current flow is in the primary circuit even if it is not currently in the secondary. The transformer is said to have no load. If the current secondary zero is zero, then the current will also be zero. However, when the converter is not loaded, the current flow flows primarily due to underlying loss and complete spinal clearance. Figure 2 shows the components of the excitation current.

Excitation current, $I_{0}$
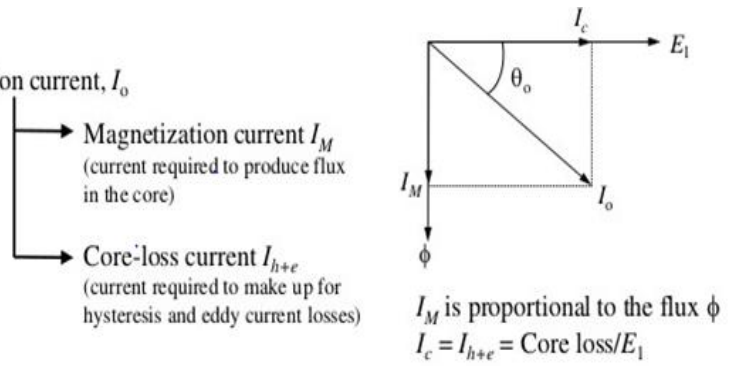

Fig. 2.Components of excitation current

\section{LINEAR CHARACTERISTICS OF MAGNETIZING CURVE}

If the applied voltage to a transformer is sinusoidal then the core flux should also be sinusoidal. If the magnetization curve of the core material would have been linear then the magnetizing current in steady state would also have been sinusoidal. Figure 3 depicts the linear characteristics of the magnetizing curve.

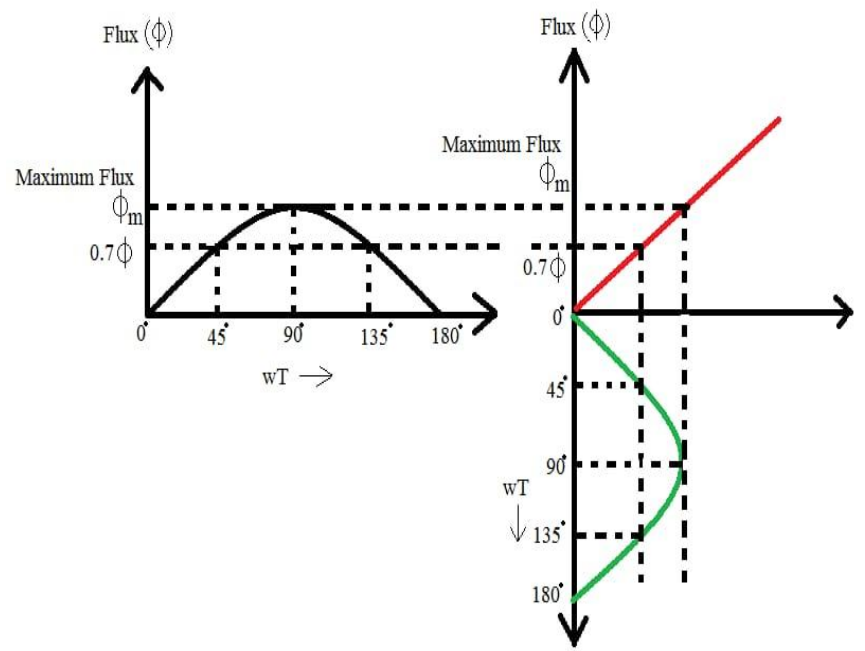

Fig. 3.The linear characteristics of magnetizing curve of core material

Published By:
Blue Eyes Intelligence Engineering and Sciences Publication

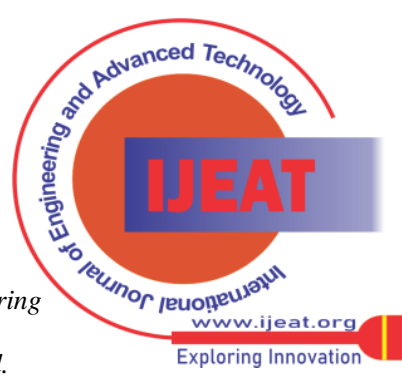


As shown in Figure 3, if the magnetic curve of core is linear then if applied voltage is sinusoidal then core flux will be sinusoidal and due to linear magnetic curve the magnetizing current in steady state will also be sinusoidal.

\section{NON-LINEAR MAGNETIZING CURVE}

Modern transformer operate with high core flux density due to economic reason and this derives the core into deep magnetic saturation with such a highly non-linear magnetizing characteristics.

Sinusoidal flux may only be obtained with a peaky magnetizing current containing dominant peaking third harmonic component if the higher order harmonics are ignored.

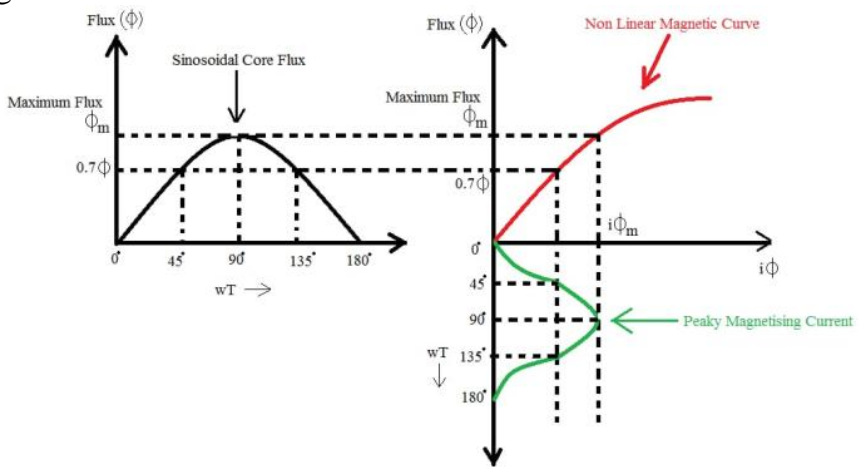

Fig. 4.Non-linear magnetizing curve of core material

In Figure 4, we will observe that if the magnetization curve of the core material is non-linear then sinusoidal flux may only be obtained with a peaky magnetizing current containing dominant peaking third harmonic.

Magnetizing current's third harmonic component may only move if electric circuit permits. Hence the third harmonic component easily flow in the single phase transformer used in single phase circuit. However, in three phase transformer connection the third harmonic component of the three phases constitute zero sequence current and therefore may only flow if the primary star neutral is connected to the source neutral or they can flow in the closed delta winding.

In star-star connection third harmonic current will not flow due to this magnetizing current is forced to remain sinusoidal

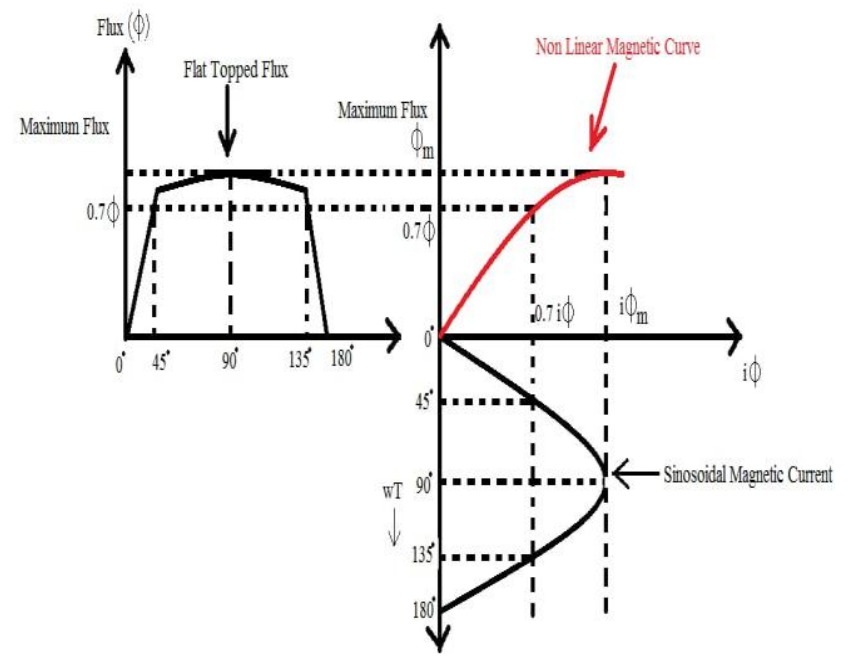

Fig. 5.The non linear magnetic curve of the core with flat topped core flux

\section{INDUCED EMF ON WINDINGS}

With a sinusoidal magnetizing current the core flux becomes flat topped containing dominant depressing third harmonic flux component constitute zero sequence flux and therefore will only get establish strongly if the magnetic circuit permits. Therefore this third harmonic flux component would easily get established in those transformers which have magnetically independent circuits, such as in three phase bank transformer , three-phase shell type transformer and five-limbed and four-limbed core type transformer. With a strong presence of third harmonic flux in star-star connected transformer having magnetically independent circuit, the induced emf of both the windings becomes peaky leading to high insulation stress besides creating a highly objectionable phenomenon called oscillating neutral.

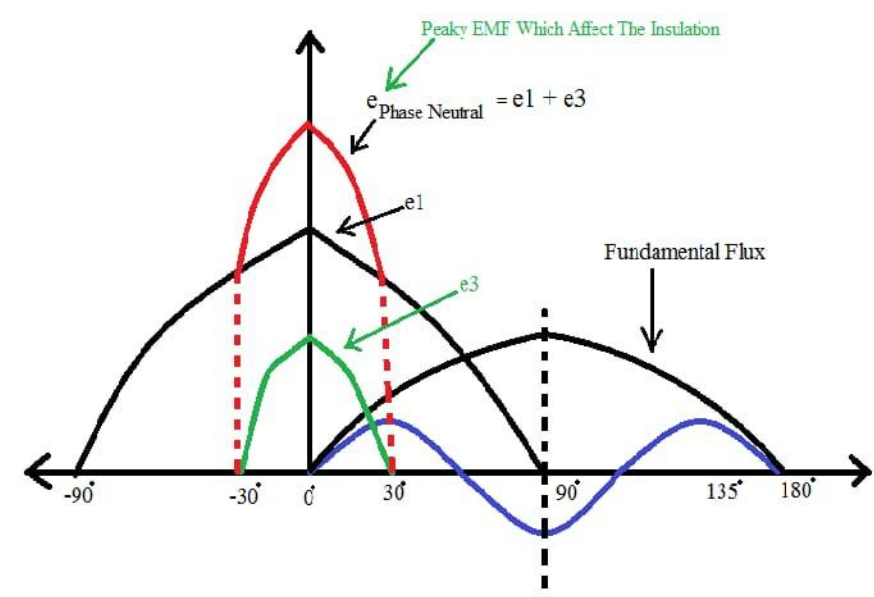

Fig. 6.The peaky induced emf of both windings

The oscillating neutral is shown in Figure 7, which comes into picture due to the third harmonic flux.

- $\quad$ The above problem of peaky induced emf of oscillating neutral is solved by providing the tertiary delta winding in star-star connection of magnetically independent circuit of transformer.

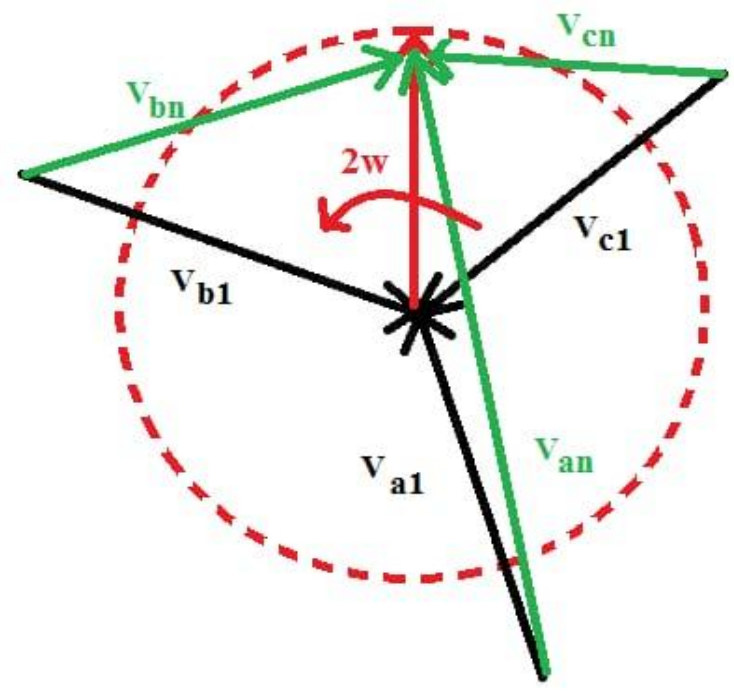

Fig. 7.Oscillating neutral

Published By:

Blue Eyes Intelligence Engineering and Sciences Publication

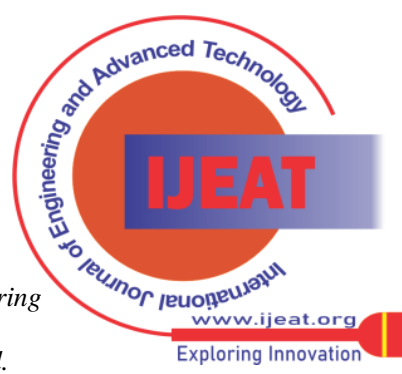


- The other option of connecting primary star neutral to generator neutral is never exercised because the generator phase voltage itself contains third harmonic voltages which would be impressed across the primary winding and get reflected into secondary winding and cause objectionable communication interference.

- In three-limbed core type transformer has magnetically interlinked circuit and therefore the third harmonic flux finds a path of very high reluctance through air and tank walls. Consequently, its strength remains negligible, hence there is a present trend to use star-star connections in three limbed core type transformers

- Absence of low reluctance path for third harmonic flux in three limbed core type transformer results into an increased component of fifth and seventh harmonic components in magnetizing current.

- Since these cannot be suppressed by any electrical connection, the only solution to prevent them is to provide a five-limbed or four-limbed core structure.

\section{CONCLUSION}

Due to the third, fifth and seventh harmonic (neglecting the higher order harmonics due to their negligible effect), it is difficult to obtain the desired emf, current or flux in the transformer. So, to avoid this. the only solution to prevent them is to provide a five-limbed or four-limbed core structure.

\section{REFERENCES}

1. R. Hamilton, "Analysis of transformer inrush current and comparison of harmonic restraint methods in transformer protection", IEEE Trans. Ind. Appl., vol. 49, no. 4, pp. 1890-1899, Jul. 2013.

2. Kartikey Singh, Amit Singh, Gautam and Lokesh Varshney, "Analysis between Various Mitigation Techniques of Inrush Current in Transformer", Presented in International IEEE Conference on Advance Computing and Innovative Technologies in Engineering, $4^{\text {th }}-5^{\text {th }}$ March, 2021

3. Lokesh Varshney, Meenakshi Sarswat, Sapna Joshi and Dilip Debnath, "Comparison between several mitigation techniques of inrush current in transformer", accepted in International IEEE Conference on Computing, Power and Communication Technologies 2018 (GUCON), $28^{\text {th }}-29^{\text {th }}$ Sep. 2018.

4. K. Karsai, D. Kerenyi, and L. Kiss, Large power transformers, Elsevier Publication, Amsterdam, 1987, pp. 41

5. A. H. A. Haj and I. E. Amin, "Factors that Influence Transformer NoLoad Current Harmonics," IEEE Transaction on Power Delivery, vol. 15, no. 1, January 2000, pp. 163-166

6. Manasi, Snigdha Sharma, Meenakshi and Lokesh Varshney, "Analysis of Equipment Sizing and Designing of 132/33/11kv Substation," Presented in International IEEE Conference on SCEECS $22^{\text {nd }}-23^{\text {rd }}$ Feb. 2020 MANIT, Bhopal.

7. Snigdha Sharma, Manasi, Meenakshi and Lokesh Varshney, "Comprehension of Different Techniques used in Increasing Output of Photovoltaic System," Presented in International Conference on Electrical and Electronics Engineering (ICEEE 2020) SPRINGER $28^{\text {th }}-29^{\text {th }}$ Feb. 2020, NPTI, Faridabad.

8. International Electrotechnical Commission Standards, IEC 76, part one, Power Transformers, 2nd ed., 1993.

\section{AUTHOR PROFILE}

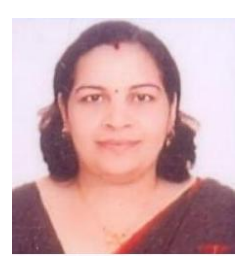

etc

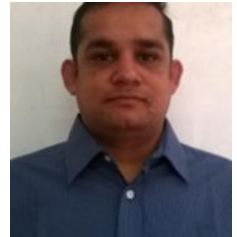

Dr. Lokesh Varshney, Received his B.E.degree from S.G.S.I.T.S, Indore. He did Both his M.Tech. and Ph.D. from IIT(BHU), Varanasi. Presently, he is working in Galgotias University, Greater Noida. His research includes Modelling of Machine,Power system etc.

Meenakshi Mataray, received the B. Tech degree in electrical in 2001 From Thiagarajar College of Engineering Madurai She has done his M. Tech in year 2008 and Now pursuing Ph.D. She has worked as Assistant Engineer in Premier Technology Chennai. Now she is working as Assistant Prof in IPEC GZB. Her research included Power System, Harmonics in Power System, Modelling of Machine

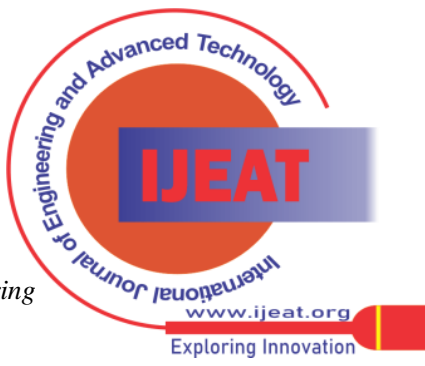

